\title{
Passivation of the CuInSe 2 surface via cadmium pre-electrolyte treatment
}

\author{
Christian Kameni Boumenou $\odot,{ }^{1}$ Finn Babbe $\odot,{ }^{1}$ Amala Elizabeth $\odot,{ }^{2,3}$ Michele Melchiorre $\odot,{ }^{1}$ Conrad Spindler,${ }^{1}$ \\ Jérome Guillot $\odot,{ }^{4}$ Harry Mönig $\odot,{ }^{2,3}$ Susanne Siebentritt $\odot,{ }^{1}$ and Alex Redinger $\odot^{1, *}$ \\ ${ }^{1}$ Department of Physics and Materials Science, University of Luxembourg, Luxembourg \\ ${ }^{2}$ Physikalisches Institut, Westfälische Wilhelms-Universität Münster, Germany \\ ${ }^{3}$ Center for Nanotechnology (CeNTech), Münster, Germany \\ ${ }^{4}$ Luxembourg Institute of Science and Technology, Luxembourg
}

(Received 15 November 2019; revised manuscript received 23 January 2020; accepted 2 March 2020;

published 15 April 2020)

\begin{abstract}
Effective defect passivation of semiconductor surfaces and interfaces is indispensable for the development of high efficiency solar cells. In this study we systematically investigated the surface and grain boundary properties of $\mathrm{CuInSe}_{2}$ (CISe) with scanning tunneling microscopy (STM) and spectroscopy (STS) after different surface treatments such as potassium cyanide $(\mathrm{KCN})$ etching, pre-electrolyte treatment with cadmium ions, and annealing in ultrahigh vacuum (UHV). We show that air exposed samples with a subsequent KCN etching step exhibits a highly defective surface. However, a Cd pre-electrolyte treatment passivates most of these defects, which manifests itself by a reduction of the high conductance in the STS measurements at positive sample biases. The origin of the improvement can be traced back to an increase in surface band bending, which leads to a type inversion, induced by a change in the concentration of $\mathrm{Cu}$ vacancies. We observe a defect passivation at the CISe surface and at the grain boundaries. Our results give a direct explanation of why the CdS buffer layer in CISe thin film solar cells is of utmost importance for high efficiency devices.
\end{abstract}

DOI: 10.1103/PhysRevMaterials.4.045405

\section{INTRODUCTION}

Polycrystalline copper indium diselenide $\mathrm{CuInSe}_{2}$ (CISe) semiconductors exhibit ideal properties for high performance thin film solar cells [1]. The absorbers exhibit a high absorption coefficient [2], a tunable direct band gap [3], high minority carrier lifetimes [4], and long diffusion lengths [4]. The solar cell devices and modules have at the same time, low manufacturing costs [5] and a small energy payback time [6]. A few years ago, the power conversion efficiencies in the laboratory surpassed the $20 \%$ efficiency benchmark due to various postdeposition treatments (PDTs) with alkali metals [7]. The surface treatments alter the chemical composition at the surface and modify the growth of the CdS buffer layer [7]. The opencircuit voltages are improved and the minority carrier lifetimes are increased $[8,9]$, which points towards a better passivation of the interface defects and/or better bulk properties.

In order to fully understand the optoelectronic modifications of the PDT treatments, a clear and concise understanding of the properties of the absorber/buffer interface prior to PDT is indispensable. Unfortunately, such a model is still not available. Photoelectron spectroscopy measurements showed that cadmium ions alter the band alignment $[10,11]$ and

\footnotetext{
*Corresponding author: alex.redinger@uni.lu
}

Published by the American Physical Society under the terms of the Creative Commons Attribution 4.0 International license. Further distribution of this work must maintain attribution to the author(s) and the published article's title, journal citation, and DOI. high resolution atom probe tomography and energy dispersive $\mathrm{x}$-ray spectroscopy [12-14] corroborated that Cd diffused into the near surface region of the absorber and substituted $\mathrm{Cu}$ vacancies, which altered the doping profile in the near surface region $[15,16]$.

Most importantly, it was shown that cadmium ions, originating from the deposition of the CdS buffer layer, play a crucial role for the fabrication of efficient devices. Especially $\mathrm{Cd}$ pre-electrolyte treatment (CPE), which is a chemical treatment that consists of $\mathrm{Cd}$ ions in an aqueous $\mathrm{NH}_{4} \mathrm{OH}$ solution, substantially improved buffer layer free devices [17]. The formation of a buried homojunction due to efficient $n$-type doping of the near surface region was suggested [18]. Theoretical calculations showed that $\mathrm{Cd}$ atoms occupying $\mathrm{Cu}$ sites [19] act as shallow donors, which explained the experimental findings. Moreover, effective doping via in-diffusion of $\mathrm{Cd}$ into the CISe lattice was reported to be facilitated by an intrinsic $\mathrm{Cu}$ depletion of the surface of $\mathrm{Cu}$-poor CISe [14,20], which has turned out to be the best composition for efficient solar cells [4]. An interesting question arises from the fact that most of these studies were done on $\mathrm{Cu}$-poor material where it is known that the surface is $\mathrm{Cu}$ depleted [14,20]. $\mathrm{Cu}$-rich absorbers, which perform worse in the final solar cell devices, exhibit less $\mathrm{Cu}$ vacancies and consequently the beneficial effect of $\mathrm{Cd}$, which is well documented for $\mathrm{Cu}$-poor absorbers, may be absent.

Since the effect of the CPE treatment is confined to the first couple of nanometers, highly surface sensitive methods need to be used. If grain boundaries need to be studied, the technique also needs to exhibit a high spatial resolution. In that respect, scanning probe techniques are ideal tools since 
they allow us to probe the electronic properties of semiconductors on the nanometer scale. In particular, Kelvin probe force microscopy (KPFM) was used frequently to study band bending at the grain boundaries [21-23]. Moreover, the effect of CPE treatment was also investigated on $\mathrm{Cu}$-poor CIGSe with KPFM and it was shown that the band bending at the grain boundaries after CPE treatment was reduced [24].

Scanning tunneling microscopy of polycrystalline CISe gave important insights into the absorber properties prior to the buffer layer deposition [25-27], such as the existence of a reduced defect density at the grain boundaries and heat induced passivation surface passivation. Large band gap fluctuations were measured with STS on epitaxially grown material [28]. In the present study we extend the STM results published so far and report how $\mathrm{Cd}$ ions alter the grain boundary and surface properties.

We concentrated on CISe absorbers without gallium and without PDTs and focused on $\mathrm{Cu}$-rich absorbers $(\mathrm{Cu} / \mathrm{In}>1)$, which are known to exhibit less potential fluctuations and better transport properties [4]. For CISe it was shown that the quasi-Fermi-level splitting on absorber layers is higher for $\mathrm{Cu}$-rich absorbers compared to $\mathrm{Cu}$-poor absorbers [29]. The drawback of $\mathrm{Cu}$-rich absorbers is that they exhibit more interface recombination, which was attributed to the absence of a Cu-depleted surface (see discussion in Ref. [4]). This paper critically discusses if the absence of $\mathrm{Cu}$ depletion is an intrinsic property of a $\mathrm{Cu}$-rich absorber and how the removal of a $\mathrm{Cu}_{x} \mathrm{Se}$ secondary phase via $\mathrm{KCN}$ etching alters the $\mathrm{Cu}$ content at the surface. As Cd doping of the CISe surface is most effective in the presence of $\mathrm{Cu}$ vacancies, we speculated that the surface of the $\mathrm{Cu}$-rich material exhibits different properties after CPE treatment.

Since STM and XPS are extremely surface sensitive, the samples need to be as clean as possible and air exposure needs to be circumvented. However, this is almost never the case since most physical vapor deposition systems that are used for CISe growth are not connected to a scanning probe system. In order to clean the surface, several studies suggested that a potassium cyanide $(\mathrm{KCN})$ treatment effectively removed the contaminations [30-32] and restored the initial quasiFermi-level splitting of the absorber [29]. Moreover, KCN etching is mandatory for $\mathrm{Cu}$-rich material in order to remove copper-selenides, which form on top of the absorber layer during growth. It was shown recently that $\mathrm{KCN}$ etching is also introducing additional defects in the near surface region that needed to be passivated with alkali elements, InSe treatments or a selenium treatment at elevated substrate temperatures [33], in order to improve the open circuit voltage of the final devices. Moreover, early work on CISe suggested that $\mathrm{KCN}$ etching may also change the surface composition via removal of $\mathrm{Cu}$ [34]. The electronic implications are not clear so far and will be discussed further in this paper.

$\mathrm{Cu}$-poor CIGSe solar cells produced with absorbers that went through a $\mathrm{KCN}$ etching step yielded the same solar cell efficiency as the ones that have not been $\mathrm{KCN}$ etched (on a 15\% device efficiency level) [21]. These results were used frequently as a justification to use $\mathrm{KCN}$ etching to produce cleaner surfaces for surface sensitive measurements [31,32] without degrading the solar cell performance [29]. The effect of $\mathrm{KCN}$ etching on device performance on $\mathrm{Cu}$-rich absorbers is difficult to access since an etching step cannot be circumvented due to the presence of $\mathrm{Cu}_{x}$ Se secondary phase after growth.

In the present study we combined scanning tunneling microscopy and spectroscopy (STS) and x-ray and ultraviolet photoemission spectroscopy (XPS/UPS) in order to link the local density of states at the surface of $\mathrm{Cu}$-rich CISe absorbers to compositional changes induced by various treatments such as $\mathrm{KCN}$ etching, cadmium pre-electrolyte treatment, and heat, and we compared our results to the already available data present in literature [24-26,35,36].

\section{EXPERIMENT}

The STM measurements were carried out in a variable temperature ultrahigh vacuum system with a base pressure in the low $10^{-11}$ mbar range. We used chemically etched tungsten tips, which were cleaned in situ by voltage pulses on a clean Au surface prior to the SPM measurements on CISe. In order to probe the local density of states at the surface we acquired current imaging tunneling spectroscopy (CITS) maps. At the region of interest (typical length scale: $\approx 1-$ $5 \mu \mathrm{m})$ a $100 \times 100$ grid was defined and local current-voltage curves were acquired while the feedback loop of the STM is switched off. The DC voltage was ramped from 1.5 to $-1.5 \mathrm{~V}$ while the current was collected. Afterwards the local density of states, which is proportional to $d I / d U$ [37], was extracted by numerical differentiation of the current with respect to the applied voltage.

Compositional measurements with XPS were carried out using an Axis Ultra DLD X-ray photo-electron spectrometer. A monochromatic $\mathrm{Al} K_{\alpha}$ X-ray source was used to illuminate the sample. The spectrometer system was calibrated with Au, $\mathrm{Cu}$, and $\mathrm{Ag}$ standards.

In order to compare the surface and bulk chemical compositions, $500 \mathrm{eV}$ sputtering with argon ions was used inside the XPS analysis chamber system. The ion fluence $(1 \times$ $10^{14}$ ions $/ \mathrm{mm}^{2}$ ) was set such that we removed approximately $5 \mathrm{~nm}$ of material. Ion erosion of a compound semiconductor is challenging due to preferential sputtering and ion induced diffusion. After sputtering we noticed a small amount of metallic indium in the XPS spectra, whereas the $\mathrm{Cu}$ and Se binding energies were unaffected (neglecting surface band bending effects). The compositions presented below do not include this metallic contribution of indium. In the Supplemental Material [38] both results are compared. All the conclusions drawn in this paper are unaffected by these differences in composition.

We also determined the valence band position after the various treatments. The samples of interest were shipped in a nitrogen filled transport box from Luxembourg to Münster where the samples were analyzed with a commercial UHV system from SPECS equipped with a PHOIBOS 100 hemispherical analyzer. We have used a $\mathrm{Al} K \alpha$ x-ray source and He I, He II UV radiation for XPS and UPS, respectively. Energy calibration was obtained by comparing the Fermi level position with measurements on clean, single, crystal metal surfaces. Throughout the paper we present the XPS measurements and a comparison to UPS measurements is included in the Supplemental Material [38]. Both techniques yielded very similar results. 
We like to emphasize that STM and x-ray photoelectron spectroscopy are very surface sensitive techniques. The STM information depth is limited to the top 2-3 monolayers, whereas the XPS and UPS is confined to approximately 10 $\mathrm{nm}$. All the conclusions drawn in the paper are therefore related to the near surface region of the absorber layers.

The CISe absorber layers studied here were grown via coevaporation on molybdenum-coated sodium-lime glass substrates at a nominal temperature of $580{ }^{\circ} \mathrm{C}$, as measured with a pyrometer. Details of growth process can be found elsewhere [39]. The CISe absorbers used in this study were air exposed after the growth for a prolonged time and were stored in a pumped desiccator. Consequently, we assumed that there was some oxidation of the surface. The thickness of the absorbers as measured using cross-sectional scanning electron microscopy was approximately $3.5 \mu \mathrm{m}$ (see the Supplemental Material [38]). To remove the copper selenide secondary phase from the surface, the samples were $\mathrm{KCN}$ etched (10 wt. \% KCN in $\mathrm{H}_{2} \mathrm{O}, 300 \mathrm{~s}$ ) and extensively rinsed with purified water in agreement with previous works [33]. We observed small holes in the absorber layer after $\mathrm{KCN}$ etching, which corroborated that the $\mathrm{Cu}_{x} \mathrm{Se}$ secondary phases were removed with the treatment (see the Supplemental Material [38]). With the water still present on the surface of the samples, they were directly introduced in a nitrogen-filled glovebox $\left(\mathrm{H}_{2} \mathrm{O}<1 \mathrm{ppm} ; \mathrm{O}_{2}<1 \mathrm{ppm}\right)$. During the purging of the load lock (cycles of pumping/refilling with $\mathrm{N}_{2}$ ) the water evaporated resulting in a surface without high amounts of oxygen and contaminations from ambient conditions. A vacuum suitcase, which can be connected to the glovebox, was used to transfer the absorber layers into the UHV SPM system. A similar procedure was followed for the XPS and PL measurements. In summary, all samples presented in this study were not exposed to ambient conditions after the chemical or heat treatments.

In CISe solar cells, the standard stacking sequence is a glass $/ \mathrm{Mo} / \mathrm{CISe} / \mathrm{CdS} / \mathrm{ZnO} /$ metal grid. Consequently, the heterojunction forms at the $\mathrm{CISe} / \mathrm{CdS}$ interface where $\mathrm{Cd}$ interdiffusion occurs, as already mentioned before. In order to study the electronic effect of $\mathrm{Cd}$, we performed the following treatment, which was based on our baseline CdS deposition recipe where $2 \mathrm{mM}$ of $\mathrm{CdSO}_{4}$ was dissolved in $1.5 \mathrm{M}$ ammonia $\left(\mathrm{NH}_{4} \mathrm{OH}\right)$. The solution is poured into a double jacketed glass reactor preheated to $67^{\circ} \mathrm{C}$. After $30 \mathrm{~s}$, the $\mathrm{KCN}$ etched samples (which were rinsed extensively with $\mathrm{H}_{2} \mathrm{O}$ ) were introduced into that solution for $3 \mathrm{~min}$. Henceforth this procedure is denoted as a Cd pre-electrolyte (CPE) treatment. Normally the next step in the recipe of the buffer layer formation would be to add a sulfur source (Thiourea) to initiate the growth of the CdS. This was not done since we were interested in the surface of the CISe, modified with $\mathrm{Cd}$ and not the $\mathrm{CdS}$ surface. Consequently, the samples were removed after the 3 min CPE treatment and were then rinsed with purified water, followed by the above described procedure to transfer them in the SPM, PL, and XPS spectrometer systems.

UHV annealing was performed as described in Ref. [26]. During the heating of the sample to $280^{\circ} \mathrm{C}$, the base pressure in the UHV chamber did not exceed $2 \times 10^{-9}$ mbar.

The photoluminescence measurements were carried out at room temperature with an argon ion laser with a laser wave- length of $660 \mathrm{~nm}$, an incident excitation power of $10 \mathrm{~mW}$, and with a spot size of $2.6 \mathrm{~mm}$. The sample were mounted on a transfer arm which was introduced into a cryostat, followed by pumping the system down to approximately $10^{-6}$ mbar. The measurements in the cryostat allowed us to carry out the measurements without air exposure.

\section{THE INFLUENCE OF Cd PRE-ELECTROLYTE TREATMENT}

\section{A. Scanning tunneling microscopy and spectroscopy measurements}

Figure 1 displays STM topography images of the same absorber after KCN etching [Fig. 1(a)] and after CPE treatment [Fig. 1(e)]. The corresponding $d I / d U$-CITS maps at different applied voltages are shown in Figs. 1(b)-1(d) for the KCN etched and Figs. 1(f)-1(h) for the CPE treated sample. All images were optimized to have the best possible contrast, as indicated by the scale bar below each subfigure.

In Figs. 1(b) $-1(\mathrm{~d})$ the $d I / d U$-CITS maps of the KCN etched samples are presented, which showed pronounced granular inhomogeneities, which manifested themselves by changes in the contrast between different grains and within individual grains. Moreover, a reduced $d I / d U$ value at the grain boundaries (GBs) compared to the grain interiors at $U=0 \mathrm{~V}$ was observed [Fig. 1(c)]. These observations were in agreement with the published results by Mönig et al. [25], who attributed the reduced $d I / d U$ contrast to a reduced defect density at the GBs. In a subsequent publication [26] the effect of UHV annealing was studied and it was found that the inhomogeneities were reduced and the distinct $d I / d U$ contrast at the GBs disappeared. We cross checked if the samples presented in this work showed the same transition and we confirmed that also here the inhomogeneities are reduced and the GB contrast disappeared after UHV annealing (see the Supplemental Material [38]). So far our measurements were in good agreement with the results reported in literature. This is somewhat unexpected, since our samples were grown under $\mathrm{Cu}$ excess, whereas most of the literature results described above were obtained on $\mathrm{Cu}$-poor material. However, in Ref. [26] Cu-poor and $\mathrm{Cu}$-rich samples were compared with STM and the authors showed that Cu-rich material exhibited less granular inhomogeneities and no reduced $d I / d U$ contrast at the grain boundaries. This is not in line with the findings presented here. However, as we will show below, the stronger $\mathrm{KCN}$ etching converted our stoichiometric surface into a $\mathrm{Cu}$-poor surface and consequently the excellent agreement between our measurements and the published results on $\mathrm{Cu}$ poor absorbers made sense. This is already a very important outcome of our study since we will show in the following that $\mathrm{KCN}$ etching is a valuable tool to form a $\mathrm{Cu}$-depleted surface, despite the fact that the growth was carried out under $\mathrm{Cu}$ excess.

In Figs. 1(f)-1(h) the changes in the local density of states after the CPE treatment are presented. We first focus on the image at $U=0 \mathrm{~V}$ [Fig. $1(\mathrm{~g})]$, which is indicative of the density of states at the Fermi level. The $d I / d U$ contrast compared to the $\mathrm{KCN}$ etched film reduced by approximately 

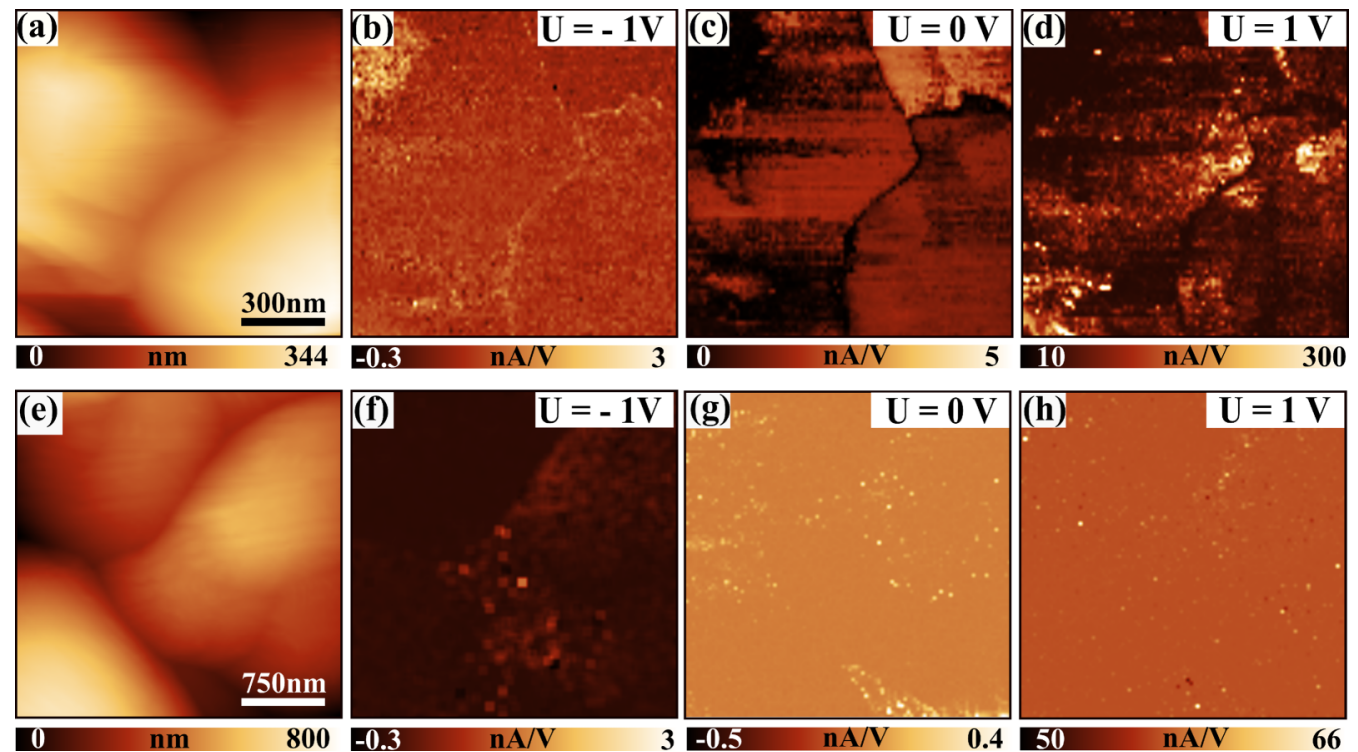

FIG. 1. (a) STM topography of a KCN etched CISe sample. (b)-(d) $d I / d U$-CITS maps of the same area at $-1,0$, and 1 V. (e) STM topography of the CPE-treated sample. (f-h) $d I / d U$-CITS maps at $-1,0$, and $1 \mathrm{~V}$. Note that the contrast of each image has been optimized as indicated by the scale bar below each image. All measurements have been carried out at the same current set point of $1 \mathrm{nA}$ at $U=1.5 \mathrm{~V}$ prior to each $d I / d U$ measurement.

a factor of 5 (see scale bar below the images), which corroborated that $\mathrm{Cd}^{2+}$ ions effectively reduced the number of defects at the surface. Moreover, the grain boundary contrast completely disappeared. At positive and negative voltages we observed some grain to grain variations [Figs. 1(f) and 1(h)]. However, the magnitude greatly reduced compared to the KCN etched case. From these measurements we asserted that the passivation effect of the CPE treatment was similar to the UHV annealed samples despite the fact that the surface treatment is very different.

The result was reproduced for a number of different spots on several samples with different tungsten tips. The extracted $d I / d U$ curves of several spots of one sample are presented in Fig. 2(a) for the KCN etched film and Fig. 2(b) for the $\mathrm{Cd}^{2+}$ treated sample. The curves in Fig. 2 represent averaged $d I / d U$ curves, i.e., an average of the $100 \times 100$ CITS map acquired for each spot. In agreement with the $d I / d U$-CITS maps presented in Fig. 1, we observed a finite conductance at the Fermi energy for the KCN etched samples, which was strongly reduced after the $\mathrm{Cd}^{2+}$ treatment (shaded region in both graphs of Fig. 2). The presented results in Fig. 1 were therefore considered to be independent of the spot chosen and generally applicable. However, we saw some scattering of the $d I / d U$ curves at positive and negative applied voltages, which we attributed mostly to changes in the work function of the tip and the sample. In the Supplemental Material [38] we discussed the reasoning for the interpretation in more detail. The important point at this stage is that there was undoubtedly a change in the density of states at the Fermi level between the $\mathrm{KCN}$ and $\mathrm{CPE}$ treated samples, which yielded a metallic $d I / d U$ curve for the KCN etched sample $\left[d I / d U\left(E_{F}\right)>0\right]$ and a semiconducting one for the CPE treated ones $\left[d I / d U\left(E_{F}\right) \approx 0\right]$. To further understand the STS data we performed XPS measurements on the same absorber layers after the different treatments.

\section{B. Surface composition as measured by XPS}

In Fig. 3 the XPS data of the $\mathrm{KCN}$ etched and $\mathrm{Cd}^{2+}$ treated samples are presented and the calculated elemental surface compositions are summarized in Table I. In Fig. 3(a) the two survey spectra are depicted and the XPS peaks of individual elements of interest are presented in Figs. 3(b)3(e). In addition to the elements in the CISe matrix, we also identified some oxygen on the absorber surfaces [Fig. 3(a)]. We attributed the oxygen to water absorption, resulting from

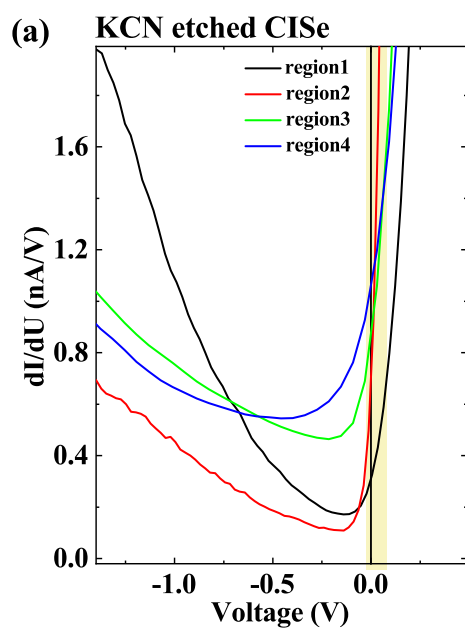

(b) CPE treated CISe

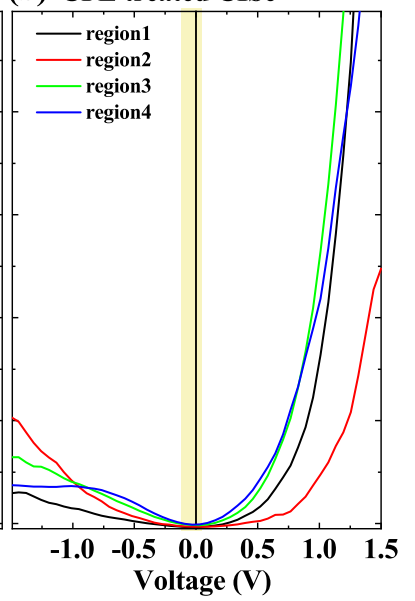

FIG. 2. $d I / d U$ curves of several spots extracted from (a) the KCN etched sample and (b) CPE treated sample. The different regions indicate that these measurements were conducted at different positions of the sample, which were at least $1 \mathrm{~mm}$ far away from each other in order to see if the results were representative. A spot denotes a microscopic region, similar to the topography images in Fig. 1, which consists of several grains including grain boundaries. 

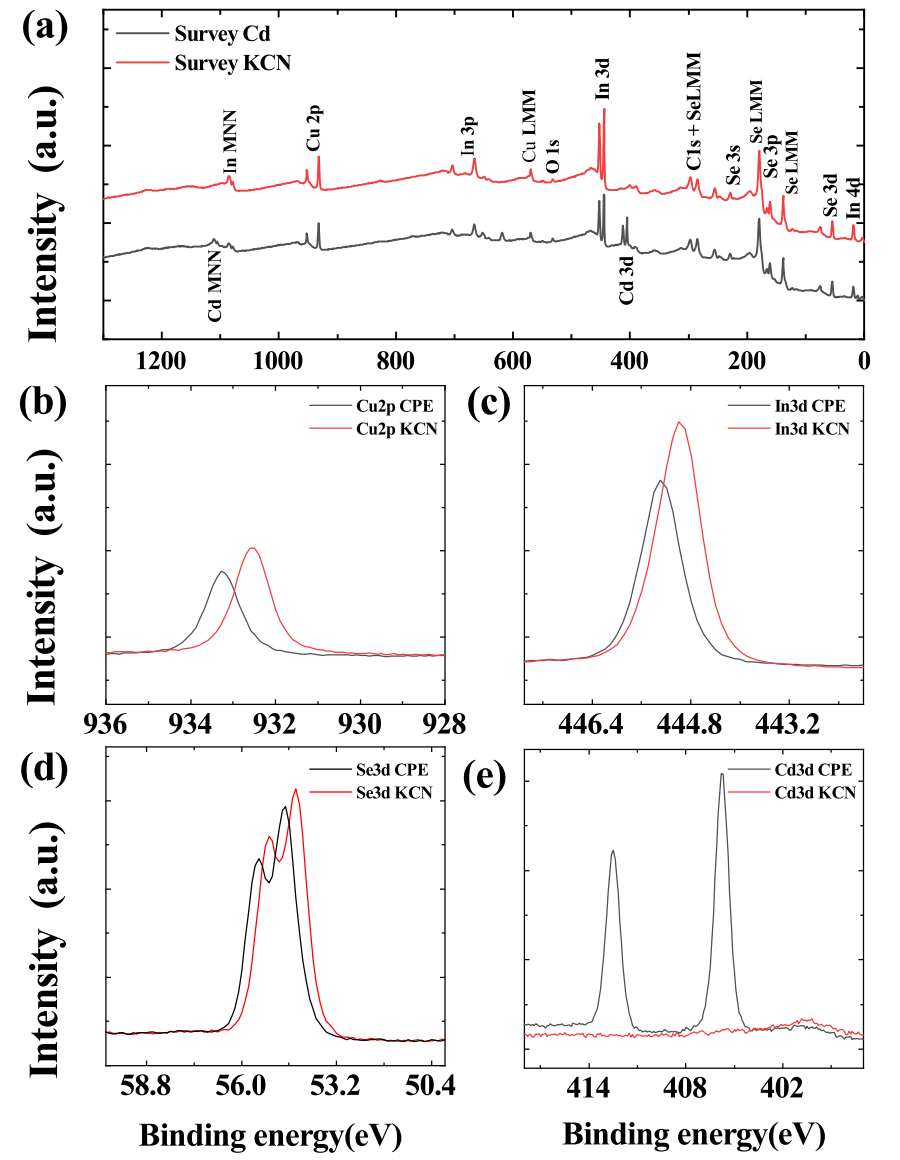

FIG. 3. (a) XPS survey spectra of the KCN and CPE treated samples. (b)-(e) Individual spectra of each element. The intensity of the $\mathrm{Cu}$ and In peaks were normalized to the In $3 d_{5 / 2}$ in order to highlight relative compositional changes.

the chemical treatment or a slight surface oxidation. The determination of the surface composition in Table I was done without taking into account the carbon signal, where the quantification is difficult due to an overlap with the selenium Auger lines. We used the $\mathrm{Cu} 3 p$ line for the quantification since this peak is closer in energy to the other elements of interest, which means that the information depth is similar. The reader is referred to the Supplemental Material [38] where we compared the changes in composition if the $\mathrm{Cu} 2 p$ is used

TABLE I. Elemental compositions of the CISe samples after various treatments, as measured via XPS. The following peaks were used for the quantification: $\mathrm{Cd} 3 d, \mathrm{Cu} 3 p$, In $3 d, \mathrm{O} 1 s, \mathrm{Se} 3 d$. The sputtering for both treatments was constant and approximately $5 \mathrm{~nm}$ of material was removed.

\begin{tabular}{lcccccc}
\hline \hline Sample & $\begin{array}{c}\mathrm{Cd} \\
(\text { at\% })\end{array}$ & $\begin{array}{c}\mathrm{Cu} \\
(\text { at\% })\end{array}$ & $\begin{array}{c}\mathrm{In} \\
(\text { at\% })\end{array}$ & $\begin{array}{c}\mathrm{O} \\
(\text { at\% }\end{array}$ & $\begin{array}{c}\mathrm{Se} \\
(\text { at\% })\end{array}$ & $\mathrm{Cu} / \mathrm{In}$ \\
\hline CISe KCN etched & 0 & 17 & 26 & 6 & 51 & 0.65 \\
Etched and sputtered & 0 & 23 & 27 & 0 & 53 & 0.84 \\
CISe Cd $\mathrm{Cd}^{2+}$ & 10 & 16 & 18 & 7 & 49 & 0.87 \\
$\mathrm{Cd}^{2+}$ and sputtered & 1 & 22 & 25 & 0 & 52 & 0.88 \\
\hline \hline
\end{tabular}

instead of the $\mathrm{Cu} 3 p$. All interpretations are independent of the choice of the Cu XPS line.

In the present case the information depth of XPS was of the order of 6-8 nm since all the XPS peaks that we used for quantification in our study are below $500 \mathrm{eV}$ in binding energy. This also means that all the changes in composition are averages over this characteristic distance.

In the following discussion we first focus on the elemental concentrations of the KCN etched sample, which showed a strong $\mathrm{Cu}$ depletion at the surface. The $\mathrm{Cu} / \mathrm{In}$ ratio was 0.65 , which was much lower than the expected value of one. After sputtering, thereby removing approximately $5 \mathrm{~nm}$ of material, the $\mathrm{Cu} / \mathrm{In}$ ratio increased $(\mathrm{Cu} / \mathrm{In}=0.84 \pm 0.04)$, which corroborated that the bulk is closer to the expected stoichiometric value of $\mathrm{Cu} / \mathrm{In}=1$. We attributed this $\mathrm{Cu}$ depletion to the strong $\mathrm{KCN}$ etching, which removed some $\mathrm{Cu}$ from the near surface region in accordance with Auger depth profiling measurements [40]. In the present case we assumed that the absolute accuracy of the compositional measurements with XPS is of the order of 1 at \%, neglecting preferential sputtering, ion beam induced diffusion, errors in the sensitivity factor, and compositional variations at the grain boundaries compared to the bulk. Taking all these simplifications into account, the measured ratio after sputtering was reasonably close to the expected stoichiometric value. After sputtering, the oxygen almost completely disappeared, which corroborated that this element was only present at the very top surface. From these measurements we concluded that our $\mathrm{KCN}$ treatment did not only remove the $\mathrm{Cu}_{x} \mathrm{Se}$ secondary phase but it also removed some $\mathrm{Cu}$ from the near surface region of the absorber. Similar observations were reported in literature [31,32], albeit less pronounced, presumably due to a different $\mathrm{KCN}$ concentration. The $\mathrm{KCN}$ etching thereby formed a $\mathrm{Cu}$-poor surface on a stoichiometric absorber, which was shown to be beneficial for device performance of stoichiometric CISe absorbers [40].

In a subsequent step our $\mathrm{KCN}$ etched films were treated with $\mathrm{Cd}^{2+}$. We observed an additional In depletion (reduction of $\approx 30 \%$ ) compared to the KCN etched sample. The result suggested that additional indium was removed from the top surface and the $\mathrm{Cu}$ content was slightly reduced [see Fig. 3(b) and Table I]. After sputtering we ended up at the same values of the $\mathrm{Cu} / \mathrm{In}$ ratio as for the $\mathrm{KCN}$ etched sample (within the error of the measurement), which showed that there was not much interdiffusion of these elements during the CPE treatment. The residual $\mathrm{Cd}$ after sputtering could arise from ion beam induced intermixing or due to some $\mathrm{Cd}$ that diffused into the bulk during the CPE, presumably along the grain boundaries in accordance with $[12,13]$. We also observed a slight reduction of the selenium concentration after $\mathrm{Cd}^{2+}$ as depicted in Fig. 3(d), although these changes are within the experimental error of the technique.

In XPS, a change in binding energy of a specific element indicates a change in the chemical environment, whereas a shift of all elemental lines is indicative of surface band bending in the case of a semiconducting surface. The XPS measurements on the $\mathrm{KCN}$ and $\mathrm{CPE}$ treated absorbers shown in Figs. 3(b)-3(e) exhibited a shift in the binding energy of all elements of approximately $300 \mathrm{meV}$, which immediately showed that there is a change in surface band bending. In 

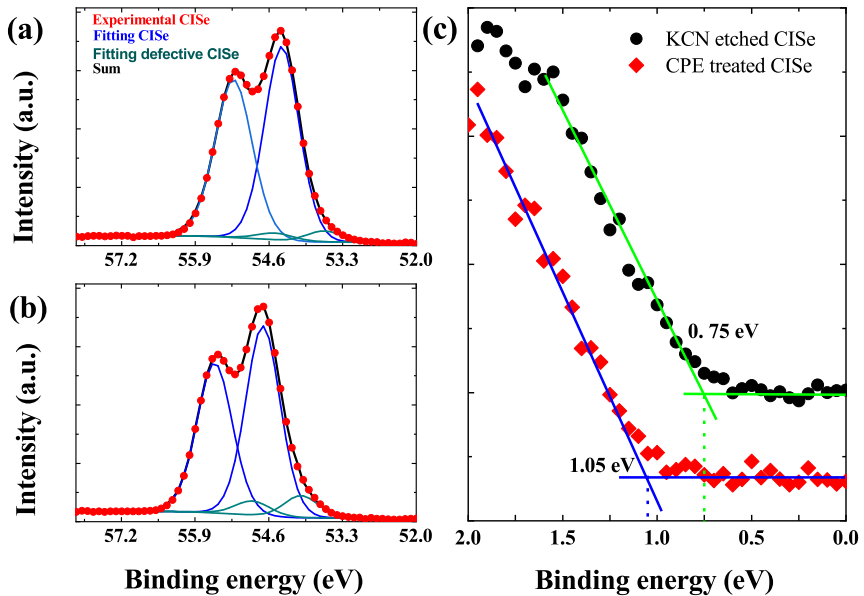

FIG. 4. (a) Se $3 d$ XPS peak of the KCN etched sample. The two main peaks correspond to the Se $3 d_{3 / 2}$ and Se $3 d_{5 / 2}$ contributions. Reasonable fitting could only be achieved if two chemical environments for the Se are used. The blue curve corresponds to the Se in the CISe matrix, whereas the green curve represents the Se atoms that are in a different oxidation state. (b) Se $3 d$ XPS peak of the CPE treated sample. The colors of all the lines are equal to (a). Note that the defective contribution is increased in the CPE case. (c) Valence band onset for the KCN and CPE treated samples measured via XPS. A comparison to UPS measurements on the same absorber can be found in the Supplemental Material [38]. The KCN etched data were shifted vertically to improve the visibility.

addition to the collective shift of the XPS peaks, we did see a change in the chemical environment of the selenium.

In Figs. 4(a) and 4(b) a peak deconvolution of the Se $3 d$ peaks is presented. The measured binding energy of $54.4 \mathrm{eV}$ is close to the tabulated values of CISe (between $54.0-54.5 \mathrm{eV}$ ) [41]. The values given here refer to the highest peak of the Se doublet, i.e., the Se $3 d_{5 / 2}$ for the KCN etched film. In the case of the KCN treated samples we found that approximately $95 \%$ of the selenium atoms are in one oxidation state (i.e., CISe), whereas 5\% exhibited a smaller binding energy (i.e., a different oxidation state). For the present discussion, we denote this peak as defective CISe since the selenium environment is distinct from the ideal case where only a single chemical environment for the Se is present. We measured a shift of $0.7 \mathrm{eV}$ to lower binding energy compared to the CISe environment. We noted that Hunger et al. [42] measured a similar peak on $\mathrm{CuInSe}_{2}$ surfaces, which were oxidized or selenium decapped. Upon $\mathrm{Cd}^{2+}$ treatment the amount of this defective peak increased substantially from approximately $5 \%$ to $10 \%$. This indicated that some of the selenium atoms in the near surface regions bound to $\mathrm{Cd}$. We noted that we also observed a slight shift of the peak position of the defective peak to higher binding energies $(\approx 0.1 \mathrm{eV})$, which corroborated that new chemical bonds formed, which were not present on the KCN etched sample. Hunger et al. [42] observed a similar change in the low energy doublet after the CPE treatment on $\mathrm{Cu}$-poor absorbers. XPS data of CdSe exhibit a peak position of $54.1 \mathrm{eV}$ [41], i.e., slightly lower than CISe, which was in accordance with our observations. The Auger parameters of both $\mathrm{In}$ and $\mathrm{Cu}$ did not change within the resolution limit of the XPS. The Auger parameter deduced from the $\mathrm{Cd} 3 d_{5 / 2}$ peak equals to $786.5 \mathrm{eV}$ in excellent agreement with the tabulated values of $\mathrm{CdSe}$ and also in agreement with Hunger et al. [42] who concluded that the majority of the $\mathrm{Cd}$ binds to the selenium atoms, presumably only in the first monolayer. They showed that $\mathrm{NH}_{4} \mathrm{OH}$ cleaning of a CISe surface selectively removed oxidized indium, which left vacancies and selenium dangling bonds at the top surface, which could then be filled with $\mathrm{Cd}$ [42]. This interpretation was in agreement with our observations where the chemical environment of the selenium atoms changed upon $\mathrm{Cd}^{2+}$ treatment, whereas the chemical environment of $\mathrm{Cu}$ and In did not change within the resolution limits of the XPS. Due to the water cleaning after the chemical treatment it was hard to disentangle residual water adsorption from indium-oxide formation on top of the $\mathrm{KCN}$ etched samples.

\section{Valence band onset for KCN and CPE treated absorbers}

Furthermore, we investigated the valence band position of the two samples with high resolution XPS at low binding energies [Fig. 4(c)]. A comparison to UPS measurements is presented in the Supplemental Material [38], which yielded very similar results. The Fermi level equals zero binding energy in the spectrum and the valence band position were deduced via linear extrapolation as shown in Fig. 4(c). We did see a shift of $0.3 \mathrm{eV}$ of the valence band position between the $\mathrm{KCN}$ etched and the CPE treated absorber. This shift is a direct consequence of band bending since we did see that all the other XPS peaks at higher binding energies exhibited a similar energetic shift. From the measurements we concluded that the CPE treated samples exhibited downward band bending. The distance between valence band and Fermi level was larger than $1 \mathrm{eV}$, which suggested that the CPE treated sample was type inverted, i.e., the majority carriers at the surface are electrons, whereas the majority carriers in the bulk are holes. We like to point out that the Fermi level was still well inside the band gap since $\mathrm{Cu}$-poor absorbers exhibit a larger surface band gap [26].

As we will see below, this type inversion had also important consequences for the interpretation of the tunneling spectra. We have also compared these results to the valence band positions after UHV annealing of the KCN etched films (the Supplemental Material [38]). We did see that after UHV annealing a similar band bending effect occurred and the value of the valence band was very close to the one after the CPE treatment $(1.08 \mathrm{eV}$ compared to $1.05 \mathrm{eV})$. This result made a lot of sense considering that we did see a very similar passivation effect of the UHV and CPE treated samples in the STM data. The XPS measurements showed that in both cases a substantial downward band bending was induced which eliminated the metalliclike conductivity in the STS spectra. The similarity between the two treatments (CPE vs UHV annealing) suggested that they had the same origin.

\section{Photoluminescence measurements}

Finally, in Fig. 5 the PL spectra of the KCN etched and the $\mathrm{Cd}^{2+}$ treated sample are depicted. The spectra are plotted on a logarithmic scale in order to better visualize small changes. We observed only a small change in intensity as a result 


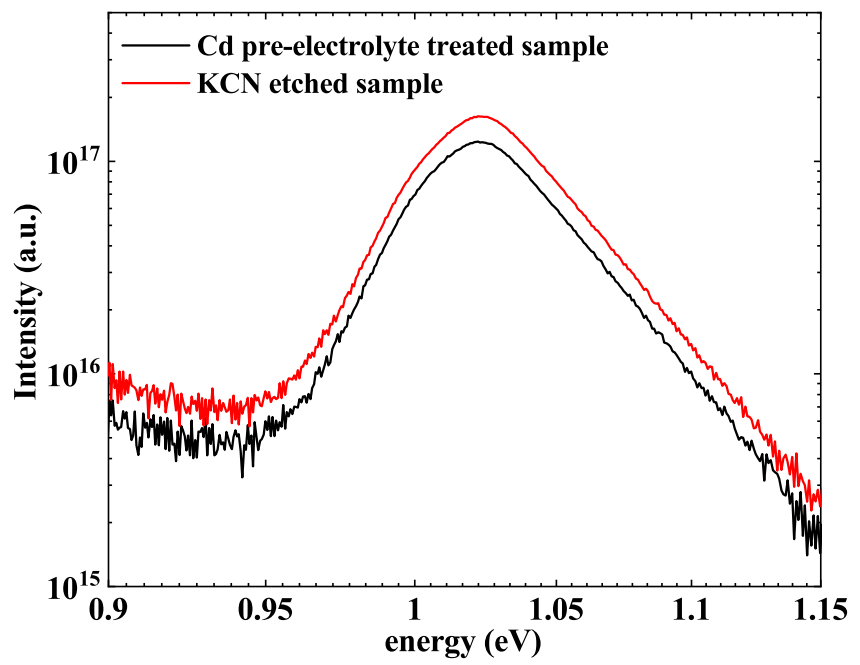

FIG. 5. PL spectra of both KCN etched and Cd pre-electrolyte treated samples. The red and dark curves were extracted from the $\mathrm{KCN}$ and $\mathrm{Cd}$ treated samples, respectively. The measurements were carried out at room temperature in an evacuated cryostat under nominally the same excitation conditions.

of slight differences in the light in/out coupling or optical adjustments. Although the samples were measured the same day with exactly the same settings without air exposure, the focus had to be re-adjusted for each sample since only one sample could be placed on the sample holder inside the evacuated cryostat. The changes in intensity by less than a factor of 2 were well within the error of the measurement. However, we need to recall measurements that were reported by Mönig et al. [35] who showed that the metallic $d I / d U$ curves could be changed to a semiconductorlike $d I / d U$ curve if the sample is illuminated with laser light. They used a laser intensity of $30 \mathrm{~mW} / \mathrm{cm}^{2}$, which was much lower than the $188 \mathrm{~mW} / \mathrm{cm}^{2}$ used in this study. Consequently, the PL measurements were not sensitive to changes in the surface state density since the high number of injected electron hole pairs screened those states very effectively. From these measurements we concluded that the surface treatment did not change the radiative recombination in the bulk of the sample and the changes in the surface state density were probably masked by the generated electron hole pairs.

\section{DISCUSSION}

In the following we construct a band diagram and discuss which defects are responsible for the observed changes in the surface density of states. Therefore, we briefly summarize the key findings of our experiments and then proceed with the interpretation:

(1) The STM measurements (Sec. III A) showed a substantial improvement of the electronic quality of the CISe surface after the $\mathrm{Cd}^{2+}$ treatment suggesting an efficient surface and grain boundary passivation.

(2) Despite the $\mathrm{Cu}$-rich growth conditions [stoichiometric bulk $(\mathrm{Cu} / \mathrm{In}=1)$ with an additional $\mathrm{Cu}_{x}$ Se on the surface], $\mathrm{KCN}$ etching led, not only to the removal of the $\mathrm{Cu}_{x} \mathrm{Se}$ secondary phase, but also to a strong $\mathrm{Cu}$ depletion right at the surface (see Table I), which gradually changed back to the stoichiometric composition after removal of a few nanometers, as shown by the XPS measurements on the sputtered CISe surface. This suggested that there is a large amount of $\mathrm{Cu}$ vacancies present in the near surface region due to $\mathrm{KCN}$ etching.

(3) The XPS measurements (Sec. III B) suggested a further reduction of the In content after the CPE treatment compared to the $\mathrm{KCN}$ etched case. This finding suggested that at least a part of the $\mathrm{Cd}$ ions was incorporated at indium sites, which was a direct consequence of the removal of indium-oxides/hydroxides in the $\mathrm{NH}_{4} \mathrm{OH}$ aqueous solution. This observation was in line with Ref. [42].

(4) XPS and UPS measurements (Sec. III C) corroborated that there was a type inversion upon CPE treatment in analogy to UHV heating. This finding showed that type inversion is a crucial ingredient to understand the STS measurements. Moreover, the CPE treatment produced the same effect as UHV annealing of KCN etched samples. The STM and the XPS/UPS data showed the same trend for both treatments.

(5) PL measurements (Sec. III D) showed that the bulk recombination is not affected by the CPE treatment. The high intensity photon flux was likely to screen most of the surface states at the surface.

The model that we propose in the following needs to be compatible with the five points mentioned above. In general, charge neutrality needs to be guaranteed at the surface and in the bulk of the semiconductor. In the presence of band bending, the charge in the space charge region $Q_{\mathrm{SC}}$ needs to be compensated by surface charges $Q_{\mathrm{S}}$, i.e., $Q_{\mathrm{SC}}+Q_{\mathrm{S}}=0$. Additionally, a dipole layer that is present at the surface may alter the local vacuum level. It is emphasized that the dipole layer itself does not contain a charge. However, the dipole layer may influence the surface chemistry, which in turn leads to a change in the number of surface charges.

We first discuss the $\mathrm{KCN}$ etched case, which is depicted in Fig. 6(a). We consider a defect denoted as $E_{\mathrm{D} 1}$, which is located above the Fermi-level $E_{\mathrm{F}}$. The defect has a certain energetic width, which is at least of the order of $k_{B} T$. In the sketch we assumed that this broadening has a Gaussian distribution. At the surface we assumed charge neutrality. Due to air exposure and subsequent etching with $\mathrm{KCN}$, the number of defects at the surface was assumed to be quite large. This is a reasonable assumption, taking into account the large compositional changes and oxygen contamination after $\mathrm{KCN}$. In order to explain our STM data, we assumed that the charge neutrality level is located above the Fermi level. This leads to the formation of positive surface charges $Q_{\mathrm{S}}$, which is compensated by a negative space charge $Q_{\mathrm{SC}}$, resulting in downward band bending. From the XPS measurements we could not measure the surface band gap and consequently we could not directly corroborate that this assumption is correct. However, other measurements on $\mathrm{Cu}$-poor materials showed that the surface band gap is approximately $1.5 \pm 0.2 \mathrm{eV}$ [26], which rendered our interpretation of a small downward band bending plausible.

Due to the broadening of the defect $E_{\mathrm{D} 1}$ the states above $E_{\mathrm{F}}$ are empty and the ones below are filled. The empty states above $E_{\mathrm{F}}$ lead to a large tunneling conductance at positive sample biases since there the current is determined by the 
(a) $\mathrm{KCN}$ etched

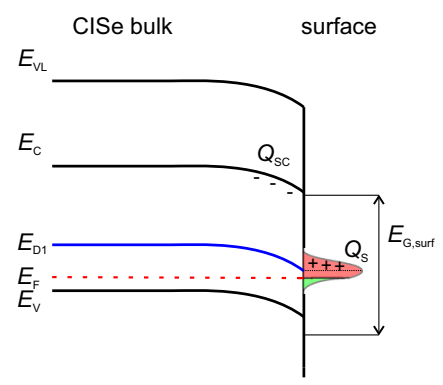

(b) CPE etched

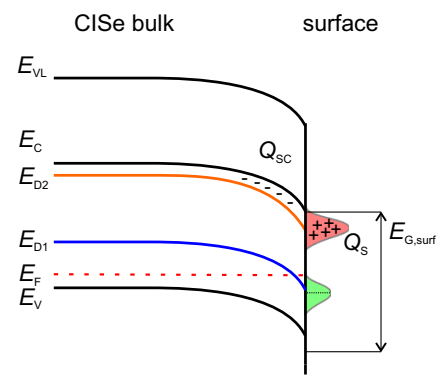

(c) after KCN

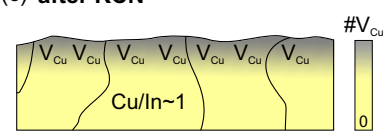

(d) after CPE-treatment

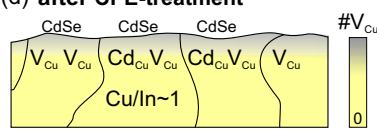

(e) after heating

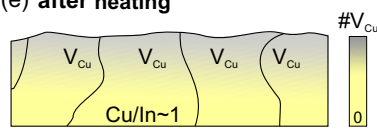

FIG. 6. (a) Band diagram of the KCN etched surface. $E_{\mathrm{VL}}, E_{\mathrm{C}}, E_{\mathrm{F}}$, and $E_{\mathrm{V}}$ denote the vacuum level, the conduction band, the Fermi level, and the valence band. The space charge is denoted as $Q_{\mathrm{SC}}$ and $Q_{\mathrm{S}}$ is the number surface charges. On KCN etched samples a large number of defects located at $E_{\mathrm{D} 1}$ gives rise to the high conductance at $E_{\mathrm{F}}$. The surface band gap is labeled as $E_{\mathrm{G}, \text { surf }}$ and is slightly larger than the bulk band gap. (b) The CPE treated sample exhibits much less defects at $E_{\mathrm{D} 1}$ and an increased number of donorlike defects at $E_{\mathrm{D} 2}$, which are empty. (c)-(e) Sketch of the evolution of the defects at the surface due to (b) $\mathrm{KCN}$, (c) $\mathrm{Cd}^{2+}$, and (d) heating in UHV. For details see text.

tunneling of electrons from the occupied tip states into empty surface states. Since the number of states right at $E_{\mathrm{F}}$ is large we do observe a finite conductance and the position of the conduction band is masked.

After the CPE treatment we observed an increase in downward band bending, which means that the number of positive surface charges needed to increase. Due to the larger band bending $E_{\mathrm{F}}$ completely crossed the defect level $E_{\mathrm{D} 1}$, as depicted in Fig. 6(b). Since the defect level thereby moved below $E_{\mathrm{F}}$, all available states at $E_{\mathrm{D} 1}$ were filled with an electron and consequently, this defect would not contribute anymore to the tunneling conductance at positive bias voltages, as in Fig. 6(a). The density of this defect needed to be much lower, as compared to the $\mathrm{KCN}$ etched case since otherwise $E_{\mathrm{F}}$ will be very close to $E_{\mathrm{D} 1}$ (i.e., Fermi-level pinning at $\left.E_{\mathrm{D} 1}\right)$. A reduction of $E_{\mathrm{D} 1}$ is however not enough to explain the experimentally observed changes. We still needed to have positive charges at the surface in order to explain the type inversion. We had two possible scenarios:

(a) In accordance with theory, we assumed that $\mathrm{Cd}$ ions, which were adsorbed at the surface during the CPE treatment, filled $\mathrm{Cu}$ vacancies. We knew from XPS that the surface is $\mathrm{Cu}$ depleted and consequently this assumption was well justified. $\mathrm{Cd}$ on $\mathrm{Cu}$ defects $\left(\mathrm{Cd}_{\mathrm{Cu}}\right)$ are known to form shallow donors [19] as shown in Fig. 6(b). Since they are located well above $E_{\mathrm{F}}$ they are empty, i.e., positively charged and induced strong band bending as observed in the XPS/UPS data.

(b) We did see that some of the In is replaced by $\mathrm{Cd}$ and probably a thin CdSe formed at the surface. This CdSe layer could be the origin of the enhanced downward band bending. In contrast to scenario (a), this did not include a change in the amount of $\mathrm{Cu}$ vacancies.

We also need to discuss the proposed passivation mechanisms in the context of UHV annealing, which had a similar effect on passivation and surface band bending in the absence of Cd ions. In Fig. 6(e) the UHV annealing is sketched. As discussed before, our absorbers exhibited a $\mathrm{Cu}$-poor surface with a stoichiometric bulk. Consequently, the heat treatment would level out some of the gradient that was present after the $\mathrm{KCN}$ treatment, i.e., the number of $\mathrm{Cu}$ vacancies were reduced. This is in analogy to the CPE treatment where the number of $\mathrm{Cu}$ vacancies reduced due to $\mathrm{Cd}$ incorporation. This interpretation is in line with the XPS measurements by Broker et al. [36] who also observed an increase in the $\mathrm{Cu}$ concentration upon UHV annealing for $\mathrm{Cu}$-rich material.

Here we show that the $\mathrm{Cd}$ treatment has a similar effect as the heat induced passivation as discussed above, by incorporating $\mathrm{Cd}$ atoms into $\mathrm{Cu}$ vacancies. From the preceding discussion it becomes clear that scenario (a) is more probable than scenario (b) since we cannot explain the passivation of the CISe via heat treatment with a CdSe layer as there is no $\mathrm{Cd}$ involved in this treatment. Therefore scenario (a) is the most probable explanation, which allows us to explain the UHV and the CPE treatment.

Finally, we would like to discuss the possible influence of the oxygen we detected on the surface of the absorber. We did not consider it to be the primary source of our observations since in all the measurements presented here, the concentrations were similar. However, this does a priori not mean that the defect concentrations are not affected by oxygen. It has been suggested by Kronik et al. [43] that an oxidized CIGSe surface exhibits no substantial band bending in contrast to an as-grown sample which always exhibits some downward band bending. We did not see any traces of oxide formation in the selenium and indium Auger lines. Consequently, we assumed that the oxygen we detect is mostly due to residual $\mathrm{H}_{2} \mathrm{O}$ and not due to an actual oxidation effect. In order to check if adsorbed $\mathrm{H}_{2} \mathrm{O}$ is responsible for the band bending and the passivation, we used a CPE treated absorber and performed a UHV annealing. This procedure removed the absorbed water and thereby the STS measurements should be modified. Since we did not observe a change in the STS measurements we ruled out that adsorbed water is responsible for the observed changes.

We still need to discuss the insensitivity of the surface treatment on the PL measurements. The electron and holes generated by the laser beam would screen the high density of surface states and consequently the changes in the surface state density would not translate in a measurable change in the PL yield. Moreover, PL essentially measures bulk properties and the change in the surface defect density will only be visible indirectly due to an increase in the surface recombination 
velocity, which needs to change by a few orders of magnitude in order to see a strong change in the PL [44].

Another important result of the present study is the change in the $d I / d U$ contrast at the grain boundaries before and after $\mathrm{Cd}^{2+}$ treatment. We could clearly show that the lower defect density at the grain boundaries after $\mathrm{KCN}$ treatment is not visible any more after the surface was exposed to $\mathrm{Cd}$ ions or heat. This extended the model proposed in Ref. [25] where the reduced defect density at the grain boundaries was identified as a characteristic of the CISe grain boundaries. The absence of a pronounced grain boundary contrast at positive voltages also allowed us to exclude a large change in work function between the grain boundaries and the grain interior. This is a direct consequence of the fact that the $d I / d U$ is proportional to the product of the density of states of the sample, tip, and the transmission function where the latter one is proportional to the work functions of the sample and the tip. The absence of a contrast at positive bias voltages is therefore a good indication that there was no band bending at the grain boundaries after the $\mathrm{Cd}$ treatment. A similar effect was observed with KPFM where the band bending also disappeared after the CPE treatment [24]. These measurements where done on $\mathrm{Cu}$-poor material, which corroborated our interpretation that the $\mathrm{Cu}$-rich and $\mathrm{Cu}$-poor surface after $\mathrm{CPE}$ treatment are electronically very similar. In both cases, $\mathrm{Cu}$-poor and $\mathrm{Cu}$-rich, the incorporation of $\mathrm{Cd}$ ions at the grain boundaries and into the near surface region of the grains led to a collective inversion of the CISe surface and the grain boundary contrast vanished.

Finally, we like to discuss the implications for solar cells in more detail. We saw that the CPE treatment led to a very similar surface for $\mathrm{Cu}$-poor and $\mathrm{Cu}$-rich absorbers and both surfaces can be passivated via an UHV annealing step. $\mathrm{Cu}$-rich absorbers produced with a $\mathrm{KCN}$ etching procedure, identical to the one discussed before, followed by a CdS buffer layer deposition, still yield inferior performance compared to
$\mathrm{Cu}$-poor absorbers [33]. This is not very intuitive since we did show that the surface of the $\mathrm{Cu}$-rich absorbers resembles in many aspects the one of $\mathrm{Cu}$-poor absorbers. However, we would like to emphasize that our observations are only valid for the first few nanometers and the absorber interior and also the grain boundaries were not affected by the CPE treatment. Consequently, much more charge carrier recombination may still be active in the bulk/grain boundaries of $\mathrm{Cu}$-rich material compared the $\mathrm{Cu}$-poor absorbers.

\section{CONCLUSION}

We showed that the surface and the grain boundary signatures of the $\mathrm{KCN}$ etched CISe surface changed significantly due to a $\mathrm{Cd}$ pre-electrolyte treatment. The grain boundary contrast vanished and the surface inhomogeneities were reduced. The positive effect of the $\mathrm{Cd}^{2+}$ treatment could be traced back to a passivation of $\mathrm{Cu}$ vacancies via $\mathrm{Cd}^{2+}$ ions. A direct consequence of the CPE treatment was an inversion of the surface in analogy to the UHV heat treatment, which was already discussed in literature. The beneficial effect of the CPE treatment is probably one of the reasons for the success of CdS in CISe thin film solar cells. Moreover, we showed that a strong $\mathrm{KCN}$ etching produces a $\mathrm{Cu}$-poor surface with a stoichiometric bulk, which was identified as one important ingredients for high efficiency CISe solar cells.

\section{ACKNOWLEDGMENTS}

This work is based upon research supported by the Fonds National de la Recherche Luxembourg (FNR), project "Sunspot," No. 11244141. A.E. and H.M. acknowledge financial support from the Deutsche Forschungsgemeinschaft through project MO 2345/5-1. Furthermore, the authors like to thank Thomas Schuler, Ulrich Siegel, Bernd Uder, and Nicolas Tournier for technical assistance.
[1] R. Scheer and H. W. Schock, Chalcogenide Photovoltaics (Wiley-VCH, Berlin, 2011).

[2] T. Kawashima, S. Adachi, H. Miyake, and K. Sugiyama, J. Appl. Phys. 84, 5202 (1998).

[3] Y. J. Jang, J. Lee, K.-B. Lee, D. Kim, and Y. Lee, J. Anal. Methods Chem. 2018, 6751964 (2018).

[4] S. Siebentritt, L. Gütay, D. Regesch, Y. Aida, and V. Deprédurand, Sol. Energy Mater. Sol. Cells 119, 18 (2013).

[5] L. Stolt, J. Hedström, J. Kessler, M. Ruckh, K. O. Velthaus, and H. W. Schock, Appl. Phys. Lett. 62, 597 (1993).

[6] T. Feurer, P. Reinhard, E. Avancini, B. Bissig, J. Löckinger, P. Fuchs, R. Carron, T. P. Weiss, J. Perrenoud, S. Stutterheim, S. Buecheler, and A. N. Tiwari, Progress in Photovoltaics: Research and Applications 25, 645 (2017).

[7] A. Chirilă, P. Reinhard, F. Pianezzi, P. Bloesch, A. R. Uhl, C. Fella, L. Kranz, D. Keller, C. Gretener, H. Hagendorfer, D. Jaeger, R. Erni, S. Nishiwaki, S. Buecheler, and A. N. Tiwari, Nat. Mater. 12, 1107 (2013).

[8] S. A. Jensen, S. Glynn, A. Kanevce, P. Dippo, J. V. Li, D. H. Levi, and D. Kuciauskas, J. Appl. Phys. 120, 063106 (2016).
[9] T. Feurer, F. Fu, T. P. Weiss, E. Avancini, J. Löckinger, S. Buecheler, and A. N. Tiwari, Thin Solid Films 670, 34 (2019).

[10] L. Weinhardt, M. Bär, H. J. Muffler, C. H. Fischer, M. C. Lux-Steiner, T. P. Niesen, F. Karg, T. Gleim, C. Heske, and E. Umbach, Thin Solid Films 431-432, 272 (2003).

[11] M. Morkel, L. Weinhardt, B. Lohmüller, C. Heske, and E. Umbach, Appl. Phys. Lett. 79, 4482 (2001).

[12] O. Cojocaru-Miŕdin, P. Choi, R. Wuerz, and D. Raabe, Appl. Phys. Lett. 98, 2 (2011).

[13] T. Nakada and A. Kunioka, Appl. Phys. Lett. 74, 2444 (1999).

[14] C. Lei, M. Duch, I. M. Robertson, and A. Rockett, J. Appl. Phys. 108, 114908 (2010).

[15] K. Ramanathan, H. Wiesner, S. Asher, R. N. Bhattacharya, J. Keane, M. A. Contreras, and R. Noufi, AIP Conf. Proc. 462, 9 (1999).

[16] F. Werner, F. Babbe, J. Burkhart, C. Spindler, H. Elanzeery, and S. Siebentritt, ACS, Appl. Mater. Interfaces 10, 28553 (2018).

[17] K. Ramanathan, F. S. Hasoon, S. Smith, D. L. Young, M. A. Contreras, P. K. Johnson, A. O. Pudov, and J. R. Sites, J. Phys. Chem. Solids 64, 1495 (2003). 
[18] P. W. Yu, S. P. Faile, and Y. S. Park, Appl. Phys. Lett. 26, 384 (1975).

[19] C. Persson, Y.-J. Zhao, S. Lany, and A. Zunger, Phys. Rev. B 72, 035211 (2005).

[20] D. Liao and A. Rockett, Appl. Phys. Lett. 82, 2829 (2003).

[21] R. Baier, C. Leendertz, D. Abou-Ras, M. C. Lux-Steiner, and S. Sadewasser, Sol. Energy Mater. Sol. Cells 130, 124 (2014).

[22] J. A. Aguiar, A. Stokes, C.-S. Jiang, T. Aoki, P. G. Kotula, M. K. Patel, B. Gorman, and M. Al-Jassim, Adv. Mater. Interfaces 3, 1600013 (2016).

[23] N. Nicoara, R. Manaligod, P. Jackson, D. Hariskos, W. Witte, G. Sozzi, R. Menozzi, and S. Sadewasser, Nat. Commun. 10, 3980 (2019).

[24] C.-S. Jiang, R. Noufi, K. Ramanathan, H. R. Moutinho, and M. M. Al-Jassim, J. Appl. Phys. 97, 053701 (2005).

[25] H. Mönig, Y. Smith, R. Caballero, C. A. Kaufmann, I. Lauermann, C. M. Lux-Steiner, and S. Sadewasser, Phys. Rev. Lett. 105, 116802 (2010).

[26] H. Mönig, D. Lockhorn, N. Aghdassi, A. Timmer, C. A. Kaufmann, R. Caballero, H. Zacharias, and H. Fuchs, Adv. Mater. Interfaces 1, 1300040 (2014).

[27] D. Azulay, D. Abou-Ras, I. Popov, I. Balberg, and O. Millo, Phys. Status Solidi 10, 448 (2016).

[28] M. A. Mayer, L. B. Ruppalt, D. Hebert, J. Lyding, and A. A. Rockett, J. Appl. Phys. 107, 034906 (2010).

[29] D. Regesch, L. Gütay, J. K. Larsen, V. Deprédurand, D. Tanaka, Y. Aida, and S. Siebentritt, Appl. Phys. Lett. 101, 112108 (2012).

[30] R. Baier, Ph.D. thesis, HZB Berlin, 2012.

[31] A. Loubat, S. Béchu, M. Bouttemy, J. Vigneron, D. Lincot, J.F. Guillemoles, and A. Etcheberry, J. Vac. Sci. Technol. A 37, 041201 (2019).

[32] J. Lehmann, S. Lehmann, I. Lauermann, T. Rissom, C. A. Kaufmann, M. C. Lux-Steiner, M. Bär, and S. Sadewasser, J. Appl. Phys. 116, 233502 (2014).
[33] H. Elanzeery, M. Melchiorre, M. Sood, F. Babbe, F. Werner, G. Brammertz, and S. Siebentritt, Phys. Rev. Mater. 3, 055403 (2019).

[34] C. D. Lokhande and G. Hodes, Solar Cells 21, 215 (1987).

[35] H. Mönig, R. Caballero, C. A. Kaufmann, T. L. Schmidt, M. C. Lux-Steiner, and S. Sadewasser, Sol. Energy Mater. Sol. Cells 95, 1537 (2011).

[36] S. Bröker, D. Kück, A. Timmer, I. Lauermann, B. Ümsür, D. Greiner, C. A. Kaufmann, and H. Mönig, ACS Appl. Mater. Interfaces 7, 13062 (2015).

[37] C. J. Chen, Introduction to Scanning Tunneling Microscopy (Oxford University Press, Oxford, 2007).

[38] See Supplemental Material at http://link.aps.org/supplemental/ 10.1103/PhysRevMaterials.4.045405 for a comparison between the UHV annealed and $\mathrm{Cd}^{2+}$ annealed $\mathrm{M}$ absorbers, a comparison between UPS and XPS results, XPS quantification for different $\mathrm{Cu}$ lines, and a discussion related to the influence of different work functions on STS spectra.

[39] F. Babbe, L. Choubrac, and S. Siebentritt, Appl. Phys. Lett. 109, 082105 (2016).

[40] V. Depredurand, Y. Aida, J. Larsen, T. Eisenbarth, A. Majerus, and S. Siebentritt, in Proceedings of the 37th IEEE Photovoltaic Specialists Conference, Seattle, WA, 2011 (IEEE, Piscataway, NJ, 2011), pp. 000337-000342.

[41] NIST X-ray Photoelectron Spectroscopy Database, NIST Standard Reference Database Number 20 (National Institute of Standards and Technology, Gaithersburg, MD, 2000).

[42] R. Hunger, T. Schulmeyer, A. Klein, W. Jaegermann, M. V. Lebedev, K. Sakurai, and S. Niki, Thin Solid Films 480-481, 218 (2005).

[43] L. Kronik, U. Rau, J. F. Guillemoles, D. Braunger, H. W. Schock, and D. Cahen, Thin Solid Films 361, 353 (2000).

[44] A. Redinger and T. Unold, Sci. Rep. 8, 1874 (2018). 